\title{
Fortalecimiento de la capacidad institucional para la equidad en la investigación en salud: enseñanzas de América Latina y el Caribe*
}

\author{
Marshall K Tulloch-Reid', Nancy Gore Saravia ${ }^{2,3}$, Rodolfo J Dennis ${ }^{4}$, Andrés Jaramillo ${ }^{5,6}$, \\ Luis Gabriel Cuervo7, Susan P Walker ${ }^{1}$ y Luis Alejandro Salicrup ${ }^{7,8}$
}

\author{
Marshall Tulloch-Reid y cols. argumentan que el desarrollo de capacidad para la investigación \\ en América Latina y el Caribe requiere inversión en instituciones tanto individuales como \\ regionales
}

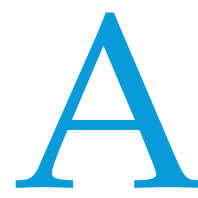

ctualmente se presta cada vez más atención a los determinantes políticos, económicos, ambientales y sociales de la salud. Esto ha generado una mayor demanda de investigación para la salud a nivel mundial que incluya a los países de ingresos bajos y medianos, con objeto de alcanzar los objetivos de desarrollo sostenible de las Naciones Unidas. ${ }^{1}$ Además del financiamiento, se necesitan suficientes recursos humanos e infraestructura para que estos países lleguen a ser asociados en un plano de igualdad con las instituciones de investigación internacionalmente reconocidas de los países de ingresos altos que establecen las agendas de investigación, y para garantizar que el programa de investigación mundial refleje sus necesidades. Las limitaciones en la capacidad de investigación y las

\footnotetext{
* Traducción oficial al español efectuada por la Organización Panamericana de la Salud. En caso de discrepancia, prevalecerá la versión original en inglés.

1 Profesor. Instituto del Caribe de Investigación para la Salud, Universidad de las Indias Occidentales, Kingston, Jamaica. $\bowtie$ marshall.tullochreid@ uwimona.edu.jm

2 Directora. Centro Internacional de Entrenamiento e Investigaciones Médicas, Cali, Colombia
}

\section{MENSAJES CLAVE}

- El desarrollo sostenible de recursos humanos requiere el fortalecimiento institucional y el desarrollo de equipos de investigación interdisciplinarios, y no solo la capacitación de científicos individuales.

- Utilizar y empoderar a las instituciones locales para que puedan adaptar la capacitación de manera que satisfaga las necesidades locales e internacionales constituye un factor fundamental en la mejora de la investigación en los países de ingresos bajos y medianos en todo el mundo.

- Se necesitan asociaciones estratégicas justas y respetuosas, junto con inversiones económicas en infraestructura, para la puesta en marcha y el mantenimiento de programas de capacitación sostenibles en los países de ingresos bajos y medianos.

- Se precisan inversiones de los organismos de financiamiento nacionales e internacionales en el desarrollo de los recursos humanos y en la creación de oportunidades para el desarrollo de una carrera de investigación, así como en la infraestructura física necesaria para la investigación para la salud.

- Se requiere una evaluación formal del éxito de la capacitación en los países de ingresos bajos y medianos de América Latina y el Caribe para demostrar mejor su efecto sobre la equidad de la investigación y la salud, y promover un mayor apoyo a estas acciones.

\footnotetext{
Directora. Universidad Icesi, Cali, Colombia

Profesor Departamento de Epidemiología Clínica y Bioestadística, Pontificia Universidad Javeriana Bogotá, Colombia

Coordinador de Proyectos de Investigación y Cursos. Centro Internacional de Entrenamiento e Investigaciones Médicas, Cali, Colombia

6 Coordinador de Proyectos de Investigación y Cursos. Universidad Icesi, Cali, Colombia
}

\begin{abstract}
Asesor Principal para la Investigación de Sistemas de Salud. Unidad de Servicios de Salud y Acceso, Departamento de Sistemas y Servicios de Salud de la Organización Panamericana de la Salud/Organización Mundial de la Salud, Washington, D.C., Estados Unidos

8 Asesor Principal para la Investigación de Sistemas de Salud Mundiales. Centro para la Salud Mundial, Instituto Nacional del Cáncer, Institutos Nacionales de Salud de Estados Unidos, Estados Unidos
\end{abstract}


grandes disparidades existentes entre los países de ingresos altos y los de ingresos bajos y medianos en cuanto a su capacidad de producir y usar la investigación para la salud han contribuido a producir una falta de evidencia que permita fundamentar la práctica clínica y orientar las políticas sanitarias regionales y mundiales. Esto ha perpetuado las desigualdades en la salud. ${ }^{2}$

En algunos países latinoamericanos ha aumentado la investigación, pero varios territorios del Caribe se encuentran entre los que tienen el nivel más bajo de producción de investigación médica. ${ }^{3}$ Mediante su Política de investigación para la salud, la Organización Panamericana de la Salud (OPS), Oficina Regional de la Organización Mundial de la Salud (OMS) para las Américas, promueve el fortalecimiento de la investigación dentro del propio país en todos los Estados Miembros, con objeto de fomentar la equidad en cuanto a la salud y el desarrollo socioeconómico. ${ }^{2}$

Describimos los resultados y las enseñanzas extraídas de tres estudios de caso, identificados por la OPS. En ellos se describen los enfoques adoptados para promover la capacidad de investigación en América Latina y el Caribe. En el primer estudio se describe la creación de capacidad para la investigación mediante la colaboración Norte-Sur y es un ejemplo latinoamericano de trabajo en red. El segundo estudio se desarrolló inicialmente mediante la cooperación bilateral seguida de la formación de redes de ámbito nacional e internacional, y se ha convertido en un modelo para la colaboración Sur-Sur. El tercer estudio de caso usa enfoques múltiples para aprovechar la infraestructura existente. Es un caso de cooperación técnica entre la OPS y el Caribe de habla inglesa.

\section{COLABORACIÓN NORTE-SUR: LA RED INTERNACIONAL DE EPIDEMIOLOGÍA CLÍNICA (INCLEN)}

En los años ochenta, la Fundación Rockefeller patrocinó la creación de la Red Internacional de Epidemiología Clínica o INCLEN para reducir la brecha existente entre la salud pública y la medicina clínica, con objeto de mejorar la salud en los países en desarrollo. ${ }^{4}$ INCLEN proporcionó becas a profesores clínicos jóvenes de facultades de medicina del sudeste asiático, China, India, África y América Latina para que recibieran capacitación en epidemiología clínica a nivel de maestría en América del Norte y Australia. ${ }^{4}$

La Fundación Rockefeller pronto se dio cuenta de que se necesitaban centros académicos de apoyo fuera de América del Norte y Australia para respaldar esta iniciativa de INCLEN. Estableció entonces asociaciones con las facultades de medicina y de salud pública locales, y brindó apoyo económico para crear unidades de epidemiología clínica, fomentando el desarrollo de conocimiento especializado en bioestadística, economía de la salud y ciencias sociales. ${ }^{4,5}$ La Fundación aportó también capacitación en gestión y liderazgo. ${ }^{4}$ A lo largo de un periodo de diez años se crearon varias unidades de epidemiología clínica, que pasaron a ser centros de excelencia para la capacitación y la investigación, con equipos capaces de dar respuesta a las necesidades de investigación regionales. Con objeto de potenciar las iniciativas de colaboración regionales, en 1991 se creó la Red de Epidemiología Clínica Latinoamericana (LatinCLEN).

La red LatinCLEN no tuvo éxito en todas partes, pero en la actualidad está presente en siete países. Las instituciones que forman parte de ella proporcionan capacitación en epidemiología clínica, bioestadística y economía de la salud, con un programa de doctorado y nueve programas de maestría. La red LatinCLEN ha abordado las necesidades regionales $\mathrm{y}$ ha llevado a cabo investigaciones con una gran repercusión en la salud mental, la salud maternoinfantil, la evaluación de economía de la salud, revisiones sistemáticas, evaluación de programas y directrices de práctica clínica (recuadro 1). A lo largo de los últimos 20 años ha tenido una influencia satisfactoria y ha liderado la cooperación con las colaboraciones Cochrane y Campbell. ${ }^{6}$

\section{COLABORACIÓN SUR-SUR: EL CASO DEL CIDEIM, UN CENTRO REGIONAL DE CAPACITACIÓN EN LA REGIÓN DE LAS AMÉRICAS}

El Centro Internacional de Entrenamiento e Investigaciones Médicas (CIDEIM) de Colombia es un buen ejemplo de asociación bilateral Norte-Sur

Recuadro 1. La Red de Epidemiología Clínica Latinoamericana (LatinCLEN)

La red LatinCLEN tiene como objetivo fortalecer los sistemas de salud nacionales y regionales mediante la investigación sobre la eficacia y la eficiencia de las intervenciones de salud y los determinantes de la salud y las enfermedades. Desde su creación, los centros de LatinCLEN han formado a más de 400 epidemiólogos clínicos y han generado más de 1200 resultados académicos. ${ }^{6}$ Los centros de LatinCLEN y sus miembros han proporcionado el liderazgo para la realización de evaluaciones económicas de las intervenciones de salud. Han perfeccionado métodos para relacionar las directrices de práctica clínica con la evaluación de economía de la salud y han establecido patrones para la evaluación de los datos económicos procedentes de diferentes fuentes. La red ha influido en las políticas de salud e investigación y en el trabajo de desarrollo de organismos y organizaciones intergubernamentales en la región.

La investigación de LatinCLEN incluye lo siguiente:

- Elaboración y validación de modelos económicos para evaluar la carga de enfermedad del tabaquismo y la relación costo-efectividad de las intervenciones para el control del tabaco en Argentina y en otros seis países en el 2011 (Grupo de Investigación en Tabaco de LatinCLEN).

- Evaluación económica de programas de rehabilitación cardíaca basados en el ejercicio físico para pacientes con insuficiencia cardiaca crónica en Colombia en el 2016.

- Determinación de la repercusión del Programa del Método Madre Canguro en Colombia.

- Evaluación de la relación costo-efectividad de nuevos medicamentos para pacientes con fibrilación auricular y síndrome coronario agudo en Chile. ${ }^{6}$ 
que evoluciona hacia un modelo para la colaboración Sur-Sur. El centro se creó en 1961 como una misión de asistencia técnica bilateral con el apoyo de una subvención a la Universidad Tulane por parte de los Institutos Nacionales de Salud de Estados Unidos. ${ }^{7}$ El centro estaba dedicado ampliamente a la investigación para la salud, y estableció una asociación con la Facultad de Medicina de la Universidad del Valle y estuvo ubicado en su recinto universitario hasta 1974. Entonces, COLCIENCIAS, el organismo nacional colombiano para la promoción y el financiamiento de la ciencia y la tecnología, asumió la codirección junto con la Universidad Tulane. El financiamiento multilateral de la investigación y las subvenciones institucionales otorgadas durante los años ochenta por COLCIENCIAS y algunos organismos internacionales sentaron las bases para la transición del CIDEIM a una institución de investigación biomédica colombiana sin fines de lucro en 1990 a través de una iniciativa pública y privada. El compromiso nacional fue crucial en cada una de las etapas de la evolución del CIDIEM. En el 2009 el CIDEIM fue elegido como centro regional de capacitación en la Región de las Américas por el Programa Especial de Investigaciones y Enseñanzas sobre Enfermedades Tropicales (TDR). Los centros regionales de capacitación colaboran en la mejora de la calidad científica y la competitividad de la investigación para la salud en los países de ingresos bajos y medianos. ${ }^{8,9}$

En el CIDEIM, los equipos interdisciplinarios diseñan y realizan investigación básica, traslacional, aplicada y de implementación, conjuntamente con las autoridades de salud pública, las universidades y las comunidades. Los proyectos en curso permiten al personal en fase de formación realizar investigación supervisada junto con los equipos de investigadores nacionales e internacionales. La investigación práctica se complementa con cursos de desarrollo de competencias, que pueden ser presenciales, de formato virtual o una combinación de ambas cosas. Los cursos incluyen temas de gestión de datos, planificación de proyectos, buenas prácticas clínicas, planificación de análisis de datos, tecnologías de la información y de la comunicación en investigación para la salud, ética de investigación e integridad científica. Este enfoque promueve la realización de la investigación cumpliendo las normas internacionales, así como la participación en las agendas de investigación nacionales e internacionales. Un aspecto importante de los cursos es su diseño orientado a que puedan ser reproducidos mediante la capacitación de instructores y la colaboración con las instituciones de las redes regionales para elaborar y adaptar módulos de capacitación.

Como centro regional de capacitación, la estrategia del CIDEIM para aumentar la capacidad se orienta a las instituciones (recuadro 2). La OPS colabora en la identificación de las instituciones participantes y aporta apoyo logístico para la difusión regional. Desde el 2009, el CIDEIM ha formado a más de 2000 investigadores, ha transferido capacidad de formación a instituciones de la red de América Central y del Sur y del Caribe, y ha llevado a cabo una sesión de capacitación interregional en colaboración con centros regionales de capacitación de Indonesia, Kazajstán y Ghana. ${ }^{10}$

\section{FORTALECIMIENTO INSTITUCIONAL MEDIANTE LA COLABORACIÓN NORTE-SUR Y SUR-SUR: UNIDAD DE EPIDEMIOLOGÍA CLÍNICA DE LA UNIVERSIDAD DE LAS INDIAS OCCIDENTALES}

La unidad de investigación en epidemiología de la Universidad de las Indias Occidentales (UWI por su sigla en inglés) se creó en 1999 como parte del Instituto de Investigación de Medicina Tropical, ahora Instituto del Caribe de Investigación para la Salud (CAIHR por su sigla en inglés). La unidad detectó la necesidad de capacitación regional en epidemiología y puso en marcha un programa de maestría en el 2005, seguido luego de un programa de doctorado.

En mayo del 2007, los participantes en la 40a reunión del Comité Asesor de Investigaciones en Salud de la OPS propusieron la formación de un equipo de epidemiólogos para aumentar la producción y el uso de la evidencia científica para fundamentar las políticas de salud pública en el Caribe. ${ }^{11}$ Los delegados de la OPS se comprometieron a apoyar el establecimiento de vínculos entre INCLEN y la UWI. La reunión fue el catalizador de una serie de avances, y la unidad de investigación en epidemiología constituyó el punto focal.

\section{Recuadro 2. Centro internacional de Entrenamiento e Investigaciones Médicas (CIDEIM)}

La capacitación es un elemento esencial en la misión y el programa de investigación del CIDEIM. La capacidad de investigación se desarrolla mediante diversos enfoques formativos. Dichos enfoques aprovechan y complementan los programas de posgrado existentes en las universidades nacionales. La investigación en curso proporciona una plataforma para llevar a cabo de manera tutelada investigación clínica, de laboratorio y sobre el terreno, en las etapas predoctoral y posdoctoral. Es importante señalar que los miembros del profesorado y los estudiantes participan en cursos de desarrollo de competencias para la investigación y en cursos de asignaturas optativas de posgrado por Internet.

Desde el 2007, se ha dispuesto de apoyo por parte del Programa Mundial de Capacitación en Investigación sobre Enfermedades Infecciosas del Centro Internacional Fogarty de los Institutos Nacionales de Salud de Estados Unidos para la realización de cursos de posgrado semestrales por Internet. Estos cursos han abordado temas avanzados de interés en biomedicina y ciencias de la salud, y han sido organizados y albergados por el CIDEIM en colaboración con la Escuela de Salud Pública Universitaria de Yale. Se realizan conferencias y debates en directo de expertos nacionales e internacionales de manera virtual, y los cursos proporcionan créditos de asignaturas optativas en los programas de posgrado de las universidades participantes. El acceso y manejo de los materiales del curso, incluidas las conferencias grabadas y las evaluaciones, se realizan con el empleo de la plataforma de docencia de código abierto Moodle. Más de 1000 investigadores y profesionales en fase de formación de 30 universidades colombianas, 12 institutos, instituciones del sector de la salud, centros de la industria y 17 universidades/institutos regionales de América Latina y el Caribe han participado en estos cursos optativos. 
Recuadro 3. Unidad de epidemiología clínica de la Universidad de las Indias Occidentales (UWI/UEC)

Mediante los puestos de capacitación destinados a candidatos doctorales de la UWI/UEC en la Universidad de Ottawa y la Universidad Johns Hopkins, se desarrolló un interés especial por las revisiones sistemáticas y la atención de salud basada en la evidencia.

Esto condujo al primer taller de capacitación en la metodología de revisión sistemática en Jamaica en marzo del 2012 (con el apoyo de profesores de la UWI/UEC y del Centro para la Salud Mundial de la Universidad de Ottawa, y de la OPS) y a la posterior puesta en marcha de la Cochrane del Caribe en junio del 2013.

La Cochrane del Caribe, junto con los miembros de la UWI/UEC, ha auspiciado los talleres anuales de capacitación Cochrane en el Instituto del Caribe de Investigación para la Salud y ha prestado apoyo técnico a los territorios del Caribe de habla inglesa. El centro también facilita los vínculos entre los investigadores locales e internacionales, e imparte capacitación sobre revisiones sistemáticas y atención de salud basada en la evidencia para estudiantes de grado y de posgrado.

La Cochrane del Caribe ha prestado apoyo para elaborar y finalizar cuatro revisiones sistemáticas iniciadas por investigadores locales, ha colaborado en más de veinte revisiones sistemáticas y ha aportado evidencia científica para la formulación de diversas políticas regionales y nacionales, de manera más reciente respecto a la política de la industria alimentaria y los formularios de medicamentos.

La unidad de epidemiología clínica de la UWI (UWI/UEC), creada en febrero del 2009, está integrada por miembros del profesorado de la unidad de epidemiología y de otros departamentos universitarios. ${ }^{12}$ Sus miembros realizaron un curso de capacitación sobre planificación y evaluación eficaces de proyectos de investigación biomédica, impartido por el CIDEIM en diversos lugares de América Latina y el Caribe. ${ }^{9}$ Una característica importante de la creación de capacidad proporcionada por el curso es la capacitación de los participantes locales para que puedan impartir el curso, lo cual permitió a la UWI repetir la capacitación en Jamaica y en otros lugares. La unidad ha proporcionado capacitación en cuanto a la planificación y evaluación de proyectos de investigación biomédica a más de 200 profesionales caribeños. La metodología se ha incorporado en cursos de posgrado de la UWI y de la Universidad de San Jorge (Granada). ${ }^{13}$ El personal de la UWI ha recibido también capacitación respecto a la síntesis del conocimiento y la traslación de la investigación a la práctica clínica. Esto facilitó la puesta en marcha de la Cochrane del Caribe en junio del $2013^{14}$ (recuadro 3).

Estas aptitudes y experiencias han ayudado a fortalecer el programa de epidemiología de la UWI. Se ha formado a 19 epidemiólogos capaces de abordar las necesidades regionales de investigación en el ámbito académico, en la administración y gestión sanitarias, y en la formulación de políticas. ${ }^{15}$ Inicialmente, muchos candidatos cursaron la capacitación para mejorar su desempeño en los puestos ya existentes o para cubrir los puestos vacantes. Actualmente se hace más hincapié en brindar oportunidades de desarrollo de investigación y trayectorias profesionales para alentar a los graduados a que permanezcan en el Caribe.

\section{RETOS EXISTENTES PARA MANTENER LA CREACIÓN DE CAPACIDAD}

El apoyo económico para mantener muchas de estas iniciativas es insuficiente. La pérdida del apoyo proporcionado por la Fundación Rockefeller ha restringido significativamente las actividades de INCLEN y LatinCLEN. La OPS respalda las iniciativas regionales de capacitación del CIDEIM y de la UWI/UEC, a través del apoyo a los talleres y los intercambios de investigadores. Sin embargo, la creación de capacidad y la formación dependen de que haya un financiamiento institucional estable y posibilidades de desarrollar una carrera profesional que atraigan a nuevas generaciones de investigadores de la salud. El personal clave de la UWI/UEC y prácticamente la totalidad de los miembros del personal del CIDEIM dependen del financiamiento de proyectos. Esto significa que la presentación de resultados de los proyectos debe equilibrarse con las actividades de creación de capacidad, y ello plantea un reto en cuanto a su sostenibilidad. Además, persiste una escasez crítica de personal capacitado en algunos campos, como los de bioestadística, ciencias de la implementación, gestión de la investigación y economía de la salud. La creación de capacidad se centra actualmente en los recursos humanos, pero hay también una necesidad fundamental de inversiones en la infraestructura de los centros y en su renovación.

\section{DISCUSIÓN}

Los estudios de caso presentados aquí muestran distintos métodos para desarrollar la capacidad de investigación en los países de ingresos bajos y medianos. ${ }^{16}$ Entre ellos se encuentran los proyectos de investigación verticales en los que los investigadores de países de ingresos altos dirigen y realizan la investigación proyectada en países de ingresos bajos y medianos; los centros de excelencia que concentran la inversión en unas pocas instituciones; las asociaciones Norte-Sur que se centran en una pregunta específica de investigación, y las redes y consorcios que establecen conexiones entre múltiples centros de investigación que disponen de capacidades complementarias para afrontar los retos de la investigación. ${ }^{16}$ En cada estudio de caso se usaron, a lo largo del tiempo, combinaciones de todos los enfoques para crear capacidades tanto individual como institucional.

Aunque los proyectos verticales pueden alcanzar los objetivos del proyecto, generalmente no producen una capacidad sostenible en los países de ingresos bajos y medianos una vez completados. INCLEN se centró inicialmente en la creación de capacidad a través de becas individuales. Sin embargo, luego encontró que el fortalecimiento de las instituciones mediante la creación de unidades de epidemiología clínica con equipos multidisciplinarios era fundamental para mantener los avances 
alcanzados en la creación de capacidad y retener el talento. ${ }^{4}$

La experiencia del CIDEIM ilustra la forma en la que las colaboraciones Norte-Sur pueden llevar al fortalecimiento de los centros de excelencia. El CIDEIM ha evolucionado a lo largo de 57 años hasta convertirse en un centro de creación de capacidad para la investigación innovadora, con influencia regional y mundial. A través de la asociación con la OPS e instituciones regionales de América Latina y el Caribe, el CIDEIM proporciona un modelo de costos compartidos para brindar capacitación para la investigación. La incorporación del contenido de cursos cortos sobre las buenas prácticas de investigación en los programas de estudio de posgrado en la región permite ampliar y mantener la creación de capacidad para la investigación.

El caso de la UWI/UEC ilustra el uso de una combinación de medidas de creación de capacidad para lograr capacidad institucional regional. El hecho de ubicar la UWI/UEC en el CAIHR, un centro que tiene como función principal la producción de investigación, ha sido fundamental al permitir a los profesionales dedicar a la investigación un tiempo específico. La OPS, junto con los asociados regionales e internacionales, al apoyar a la UWI/UEC en la formación de un equipo de epidemiólogos regionales en el Caribe, ha permitido alcanzar una repercusión más amplia y más sostenible. ${ }^{13}$

Las limitaciones actuales en el financiamiento nacional e internacional de la investigación no fomentan la inversión en la creación de capacidad local en los países, para lo cual se necesitan muchos años de apoyo sostenido. Las redes de investigación de América Latina y el Caribe siguen buscando colaboraciones Norte-Sur para el financiamiento. En una revisión sistemática reciente que examinó el desarrollo de capacidades para la investigación, se observó que la mayor parte de la investigación basada en las colaboraciones Norte-Sur se centraba en los problemas existentes en esas relaciones. Muchas publicaciones argumentan que los beneficios que obtiene el asociado del Sur son demasiado escasos. ${ }^{16}$

Varias organizaciones mundiales han instado a que la creación de capacidad sea un componente clave del financiamiento de la investigación mundial, con objeto de reducir la "fuga de cerebros" de los países de ingresos bajos y medianos. ${ }^{17}$ La existencia de oportunidades estables para el desarrollo de una carrera profesional en investigación constituye un determinante clave de la capacidad de investigación y de la formación en investigación. Los sistemas de financiamiento para la creación de capacidad podrían aprovechar los puntos fuertes ya existentes en las instituciones de América Latina y el Caribe. Un sistema de financiamiento posdoctoral para los países de ingresos bajos y medianos podría empezar a abordar esta etapa de transición fundamental en el desarrollo profesional, en particular en esta región en donde la mayoría de los países disponen de programas de capacitación de posgrado.

Algunos organismos internacionales han instituido sistemas de financiamiento dirigidos a equipos de investigación de los países de ingresos bajos y medianos. Entre ellos se encuentran los sistemas de subvenciones de los Centros de Investigación Internacional en Enfermedades Infecciosas y Medicina Tropical de los Institutos Nacionales de Salud de Estados Unidos y los programas de capacitación Fogarty. El Wellcome Trust ofrece diversos programas de becas internacionales, proyectos colaborativos e incluso subvenciones para equipamiento multiusuario. ${ }^{18}$

Los centros de países de ingresos bajos y medianos, en particular los de América Latina y el Caribe, tienen que mejorar su capacidad de proporcionar proyecciones exactas y congruentes de los costos totales de la investigación a los organismos de financiamiento y de obtener un reembolso adecuado de los costos indirectos. ${ }^{20,21}$ Algunos organismos de financiamiento reconocen las restricciones impuestas por la limitación del apoyo aportado para los gastos de ejecución y están dispuestos a negociar costos indirectos mayores para esos centros; sin embargo, otros ponen un límite a estos recursos en un valor inferior al que se aplica en los países de ingresos altos. ${ }^{19}$ Una limitación importante en los costos indirectos tiene una repercusión negativa en el apoyo administrativo y los gastos de capital en infraestructura y equipo, lo que desincentiva la participación de investigadores y profesores en actividades de capacitación sin ánimo de lucro. Los centros de países de ingresos bajos y medianos necesitan este apoyo para tener reconocimiento como puntos focales creíbles para la creación y el mantenimiento del cambio. ${ }^{20}$

Las soluciones nacionales y regionales para el financiamiento de los centros de América Latina y el Caribe incluyen la inversión en su propia creación de capacidad, mediante la dedicación de una parte de los presupuestos nacionales o de impuestos especiales a financiar la investigación y la infraestructura de investigación. También es posible la colaboración con los científicos de la diáspora nacional de la investigación. ${ }^{17}$ Además de abordar los problemas de salud urgentes, mejorar la evaluación del papel fundamental que desempeñan los centros de investigación en el desarrollo nacional puede alentar a los responsables de formular políticas a que reconozcan la investigación para la salud como una inversión en lugar de como un gasto. ${ }^{2}$

\section{CONCLUSIONES}

A pesar de los diversos retos que plantea la creación de capacidad para la investigación en los países de ingresos bajos y medianos, los tres estudios de casos presentados aquí ponen de manifiesto que es posible obtener resultados positivos mediante el uso coordinado de las redes existentes y los limitados fondos disponibles. También destacan la importancia de la colaboración Norte-Sur y Sur-Sur para aumentar la capacidad para la investigación con objeto de mejorar la salud en toda la Región de las Américas. Algunos componentes clave de su éxito han sido prestar apoyo a líderes comprometidos, proporcionar capacitación aprovechando las iniciativas de capacitación regionales existentes, y crear buenas asociaciones regionales e internacionales. La presencia de centros de investigación capaces en América Latina y el Caribe será crucial para que la investigación para la salud sea sostenible y equitativa en el futuro.

Contribución de los autores. Todos los autores han liderado o facilitado muchas de las iniciativas descritas en este artículo y han participado en la conceptualización, redacción y revisión del texto, han aprobado la versión definitiva del artículo presentado para publicación y son responsables de todas las partes del trabajo en que han participado. MKT-R es el garante del artículo.

Conflicto de intereses. Hemos leído y comprendido la política de The BMJ sobre el conflicto de intereses y no tenemos intereses pertinentes que declarar. 
Procedencia del artículo y revisión por pares. Comisionado; revisión por pares externa.

Declaración. Las opiniones expresadas en este manuscrito son responsabilidad del autor y no reflejan necesariamente los criterios ni la política de la RPSP / PAJPH y/o de la OPS/OMS.
Serie Fortalecimiento de la investigación para la salud en las Américas. Este artículo es parte de una serie propuesta por la Organización Panamericana de la Salud (OPS) y comisionada por The BMJ, que tuvo a cargo la revisión por pares y la edición, y tomó la decisión sobre la publicación de los artículos sin participación de la OPS. Los

\section{REFERENCIAS}

1. Organización Mundial de la Salud. Subsanar las desigualdades en una generación: Alcanzar la equidad sanitaria actuando sobre los determinantes sociales de la salud. Comisión sobre Determinantes sociales de la salud - Informe Final. 2008. https:// www.who.int/social_determinants/the commission/finalreport/es/

2. Organización Panamericana de la Salud/ Organización Mundial de la Salud. Política de investigación para la salud. Documento CD49/10, $49 .{ }^{\circ}$ Consejo Directivo de la OPS. 2009. https://www.paho.org/hq/index .php?option $=$ com_content\&view $=$ article\& id $=1414 \% 3 \mathrm{~A} 2009$-policy-on-research-healt h\&catid $=2502 \% 3$ Apublications\&lang=en

3. McKee M, Stuckler D, Basu S. Where there is no health research: what can be done to fill the global gaps in health research? PLoS Med 2012;9:e1001209. http://journals .plos.org / plosmedicine / article?id $=10$ .1371/journal.pmed.1001209. doi:10.1371 /journal. pmed.1001209

4. Halstead SB, Tugwell P, Bennett K. The International Clinical Epidemiology Network (INCLEN): a progress report. J Clin Epidemiol 1991;44:579-89. doi:10 .1016/0895-4356(91)90222-U

5. Morrow RHJr, Lansang MA. The role of clinical epidemiology in establishing essential national health research capabilities in developing countries. Infect Dis Clin North Am 1991;5:235-46.

6. Gómez Restrepo C, Muñoz N S, Ruiz AJ, Lanas F. Latin American Clinical Epidemiology Network Series - Paper 1: The Latin American Clinical Epidemiology Network "LatinCLEN". J Clin Epidemiol 2017;86:714. doi:10.1016/j.jclinepi.2016.10.002

7. Board on Science and Technology for International Development, Institute of Medicine National Academy of Sciences. The US capacity to address tropical infectious disease problems. National Academy Press, 1987. https:/ / books.google.co.uk/books?id=C pYrAAAAYAAJ\&pg $=$ PR2\&lpg $=$ PR2\&dq $=$ The + US + Capacity+to+Address + Tropica 1+Infectious+Disease+Problems.+Nationa
1+Academy+of+Science+1987.+Report+No .\&source $=$ bl\&ots $=$ HsxQz52EoX\&sig $=C$ b5u1-65MEE4PTSqcpTo_d4Y0MY\&hl=en\& sa $=X \&$ redir_esc $=y$

8. Organización Mundial de la Salud. Regional training centers. An introduction to the TDR network. 2015. www.who.int/entity /tdr/news/2015/en/

9. Ogundahunsi OA, Vahedi M, Kamau EM, et al. Strengthening research capacityTDR's evolving experience in low- and middle-income countries. PLoS Negl Trop Dis 2015;9:e3380. doi:10.1371/journal. pntd.0003380 http://journals.plos.org / plosntds / article?id=10.1371/ journal .pntd.0003380.

10. Alger J, Gómez L, Jaramillo A, Saravia NG, Cuervo LG. Reunión de la Red Inter-Regional de Planeación y Evaluación Efectivas de Proyectos de Investigación para la Salud, Cali, Colombia, Abril del 2010. Rev Med Hondur 2010;78:97-100. http: / / www.paho.org/hq/index.php ?option $=$ com_docman\&task $=$ doc_view $\&$ gid $=17086 \&$ Itemid $=270 \& l a n g=e s$

11. Comité Asesor de Investigaciones en Salud (CAIS). Organización Panamericana de la Salud/Organización Mundial de la Salud (OPS/OMS). $40^{\circ}$ CAIS: Panorama de la situación de las políticas de investigación en las Américas y propuesta para el desarrollo de una política en investigación en salud de la Organización. 2007. http: / / www1.paho.org/spanish/DD /IKM / RC / CAIS-2007-40-05-Panorama pdf?ua $=1$

12. Universidad de las Indias Orientales. UWI admitted to International Epidemiology Network. Nota de prensa. https://www .mona.uwi.edu/marcom/uwinotebook /entry/3492

13. Organización Panamericana de la Salud/ Organización Mundial de la Salud. Report on strengthening research capacities for health in the Caribbean, 2007-2017. Washington, DC: OPS/OMS, 2017. http:// iris.paho.org/xmlui/handle/123456789 /34342 gastos de publicación en acceso abierto de la serie original en inglés fueron financiados por la OPS. Acceso a la serie original en inglés en www.bmj.com /health-research-americas; acceso a la serie en español y portugués en https:/ / www.paho.org/journal/es/numeros -especiales/fortalecimiento-investigacion -para-salud-americas.
14. Cochrane Caribbean. Cochrane Caribbean launch. Nota de prensa, 2013. http:/ / caribbean.cochrane.org/cochrane -caribbean-launch

15. Tulloch-Reid MK. The University of the West Indies Clinical Epidemiology Unit: collaborating to improve health. J Clin Epidemiol 2015;68:1099-100. doi:10.1016/j. jclinepi.2015.04.012

16. Franzen SR, Chandler C, Lang T. Health research capacity development in low- and middle-income countries: reality or rhetoric? A systematic meta-narrative review of the qualitative literature. BMJ Open 2017;7:e012332. doi:10.1136/bmjopen-2016 $-012332$.

17. Saravia NG, Miranda JF. Plumbing the brain drain. Bull World Health Organ 2004;82:608-15.

18. Wellcome Trust. Scheme finder. Reino Unido: Research Council, 2018. https:// wellcome.ac.uk/funding/scheme-finder

19. Institutos Nacionales de Salud de Estados Unidos. NIH grants policy statement. 2017. https://grants.nih.gov/grants/policy /nihgps/html5/section_7/7.4_reimbursement_of_facilities_and_administrative _costs.htm

20. Lucas S. How to fund think tanks effectively even when you can't. William and Flora Hewlett Foundation, 2017. https: / / www.hewlett.org/fund-think -tanks-flexibly-even-cant/

21. Consejo de Investigación en Salud para el Desarrollo. Fair research contracting. Ginebra (Suiza), 2018. http:/ / www.cohred .org/frc/

Forma de citar (artículo original): BMJ 2018;362:k2456 doi: http:/ /dx.doi.org/10.1136/bmj.k2456

Manuscrito original en inglés publicado el 16 de julio del 2018. 